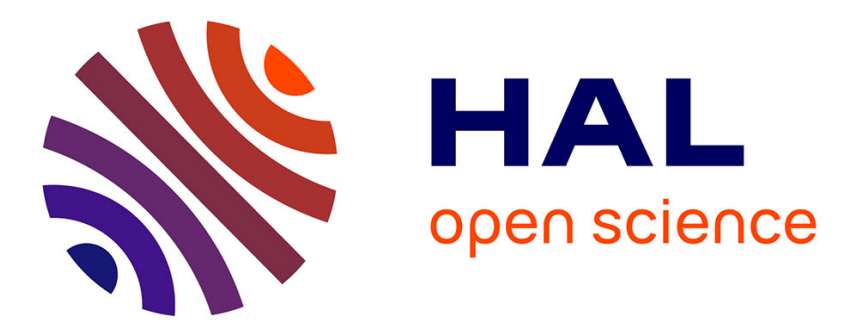

\title{
Automatic segmentation of pathological tissues in cardiac MRI
}

Khaoula Elagouni, Cybèle Ciofolo-Veit, Benoît Mory

\section{To cite this version:}

Khaoula Elagouni, Cybèle Ciofolo-Veit, Benoît Mory. Automatic segmentation of pathological tissues in cardiac MRI. 2010 IEEE International Symposium on Biomedical Imaging: From Nano to Macro, Apr 2010, Rotterdam, Netherlands. pp.472-475, 10.1109/ISBI.2010.5490306 . hal-00508765

\section{HAL Id: hal-00508765 https://hal.science/hal-00508765}

Submitted on 5 Aug 2010

HAL is a multi-disciplinary open access archive for the deposit and dissemination of scientific research documents, whether they are published or not. The documents may come from teaching and research institutions in France or abroad, or from public or private research centers.
L'archive ouverte pluridisciplinaire HAL, est destinée au dépôt et à la diffusion de documents scientifiques de niveau recherche, publiés ou non, émanant des établissements d'enseignement et de recherche français ou étrangers, des laboratoires publics ou privés. 


\title{
AUTOMATIC SEGMENTATION OF PATHOLOGICAL TISSUES IN CARDIAC MRI
}

\author{
Khaoula Elagouni ${ }^{1}$, Cybèle Ciofolo-Veit ${ }^{1}$, Benôt Mory ${ }^{1}$ \\ ${ }^{1}$ Philips Medical Systems Research Paris, Suresnes Cedex, France.
}

\begin{abstract}
In the context of cardiac viability assessment, we propose a new fully automatic method to segment and quantify myocardial pathological tissues in Late Enhancement Cardiac Magnetic Resonance images. Our two main contributions are a generic image intensity analysis and an original variational segmentation method, the Fast Region Competition. The obtained results are robust to anatomical variability and partial volume effects and false positives are avoided. To validate our results, we use representations that are independent of myocardium shape and size and compute clinically relevant indicators. The proposed method was tested on 100 slices and compared to other classical segmentation approaches, showing the best agreement with semi-automatic expert delineations.
\end{abstract}

Index Terms - Segmentation, variational methods, lateenhancement, myocardium viability.

\section{INTRODUCTION}

Viability assessment of the heart muscle is essential for diagnosis and therapy planning. It is based on clinical measurements of pathological tissues, which can be efficiently visualized with Magnetic Resonance Imaging (MRI). The Late Enhancement Cardiac Magnetic Resonance (LECMR) protocol consists of acquiring images around twenty minutes after a contrast agent injection. It provides a stack of 10 to 12 slices on which abnormal tissues (for example, infarcted areas) appear brighter than normal myocardium (see Fig. 1). In this work, we focus on the automatic and robust detection of pathological regions with the final objective to derive relevant clinical parameters for viability quantification.

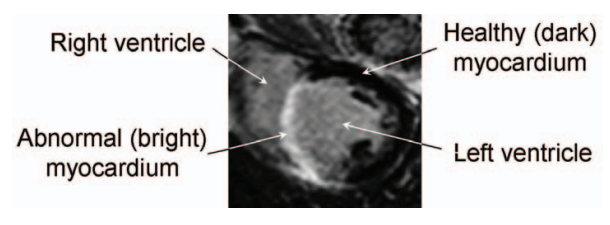

Fig. 1. Sample LE CMR slice

To this end, several issues have to be solved. First, depending on the pathology, the shape and angular extent of ab- normal areas, their localization with respect to myocardium contours and the sharpness of their borders vary a lot. Moreover, the contrast between normal and abnormal tissues is related to the acquisition time, which is not always optimal, making the segmentation difficult and prone to intra and interobserver variability.

These reasons motivate the use of a robust and automatic segmentation algorithm. In this context, the most intuitive idea is to threshold image intensities. Different works propose to automatically compute the best threshold and characterize hyper-enhanced regions $[1,2,3]$. The main drawback of these methods is the lack of spatial coherence, leading to numerous false positives or, on the contrary, under-segmented pathological regions. To improve this, Hsu alternates thresholding and feature analysis [4], assuming that abnormal tissues are mainly sub-endocardial and reasonably large. Heiberg introduces a level set algorithm to regularize thresholding [5], while in Hennemuth's recent approach, the threshold resulting of image intensity analysis is used to initialize a watershed segmentation [6]. However, besides the spatial coherence brought by all these methods, it is always necessary to use a connectivity-based post-processing to refine the results. In this paper, we propose a new method with two main contributions: (1) the generalization of the intensity analysis presented in [6] and (2) an original variational segmentation method, the Fast Region Competition, leading to accurate results without any assumption concerning the location of abnormal tissues nor need for post-processing.

\section{DETECTION AND SEGMENTATION}

Assuming that the myocardium contours have already been automatically obtained [7], our automatic segmentation method consists of four steps, as illustrated in Fig. 2. First we analyze myocardium intensities and estimate normal and pathological tissues distributions. These distributions are used to generate a fuzzy map indicating, for every pixel, the membership degree to the abnormal tissue class. After fuzzy map thresholding and morphological cleaning, the Fast Region Competition is applied to precisely segment pathological tissues. The fuzzy map is finally superimposed on the segmentation results to visualize pathological tissue severity. 


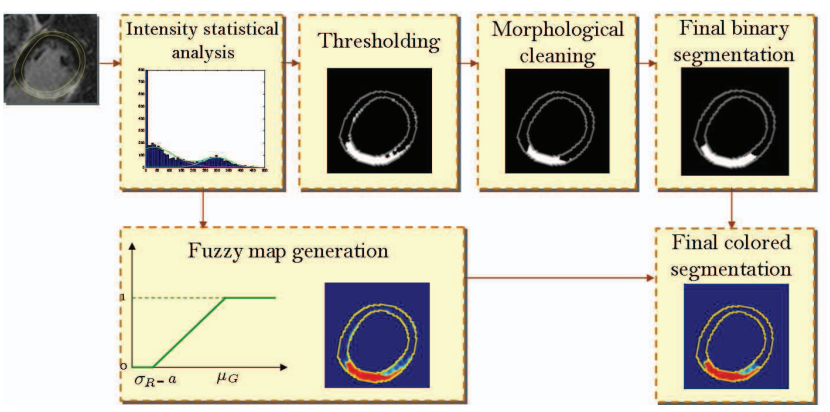

Fig. 2. Pathological tissues detection scheme.

\subsection{Statistical intensity analysis}

In the presence of noise, the magnetic resonance image intensity is shown to be governed by a Rician distribution [8], which reduces to a Rayleigh distribution when intensity values are close to zero, and tends to a Gaussian distribution when values are high. In the case of LECMR and inside the myocardium, the Rayleigh and the Gaussian distributions adequately represent respectively normal (dark regions) and pathological tissues (bright regions). In order to fit a Rayleigh-Gaussian mixture to the image histogram, we express the distribution function related to myocardium as follows:

$$
p_{\text {myoc }}(I)=\alpha_{R} \cdot p_{R}\left(I, \sigma_{R}\right)+\alpha_{G} \cdot p_{G}\left(I, \mu_{G}, \sigma_{G}\right),
$$

where $\alpha_{R}$ and $\alpha_{G}$ are distributions weights, and $\sigma_{R}, \mu_{G}$, and $\sigma_{G}$ are distributions parameters.

Some signal processing is applied during the acquisition to view the normal myocardium as dark as possible and help abnormal tissue identification. Consequently, the intensities are often shifted to the left with an unknown offset $a$ whose value depends on acquisition parameters. Ignoring this offset may lead to inaccurate intensity distribution analysis and incorrect segmentation of abnormal regions, as illustrated in Fig.3. Our first contribution is thus to introduce $a$ as an additional parameter in the estimation. However, we assume that it is small enough to ignore the shifted part of the Gaussian distribution. Eq. (1) can then be written as:

$$
p_{\text {myoc }}(I)=\alpha_{R} \cdot p_{R}\left(I+a, \sigma_{R}\right)+\alpha_{G} \cdot p_{G}\left(I, \mu_{G}, \sigma_{G}\right) .
$$

The estimation of the distribution parameters can be formalized as maximizing the likelihood between a set of observations, namely the myocardium histogram, and a RayleighGaussian mixture model. This is done with an ExpectationMaximization (EM) algorithm [9] that we adapted to recover the additional offset parameter $a$. In particular, the analysis of the Rayleigh distribution properties leads to:

$$
\sigma_{R}=\frac{\int_{I_{M}}^{\infty} p_{R}(t) \cdot d t}{p_{R}\left(I_{M}\right) h\left(I_{M}\right)},
$$

where $I_{M}$ is the intensity corresponding to the histogram maximum and $h\left(I_{M}\right)$ its number of occurences, which immediately yields $a=\sigma_{R}-I_{M}$. These relationships are introduced in the maximization step of the EM algorithm to obtain the distributions representing normal and pathological tissues (see Fig. 3).

The distributions are then exploited to generate a fuzzy map indicating, for every pixel, the membership degree to the abnormal tissues class. To do so, we apply a ramp model such as the membership value is 0 if $i \leq \sigma_{R}-a, 1$ if $i \geq \mu_{G}$ and varies linearly between these bounds. An example of fuzzy map is visible in Fig. 4(B).

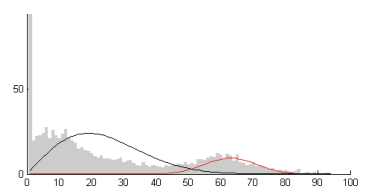

(A) Ignoring parameter $a$

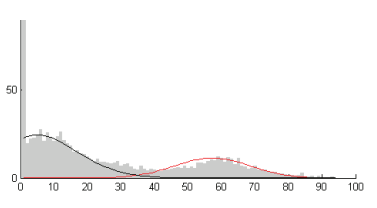

(B) Estimating parameter $a$
Fig. 3. Intensity analysis: influence of the offset $a$ parameter. Black: estimated Rayleigh distribution (healthy tissue), red: estimation Gaussian distribution (abnormal tissue).

In order to detect abnormal regions, we first threshold the fuzzy map, keeping only pixels whose membership value is over 0.5 . The reason for using an arbitrary threshold instead of computing the distributions intersection is that, for small scars, the abnormal tissue distribution may be underrepresented. In this case, the distributions intersection is higher than the mean of the Gaussian distribution, which is not a physiologically acceptable threshold, contrary to the 0.5 membership value. Moreover, the segmentation method that follows is not sensitive to the threshold value, provided that it is chosen between the distributions peak values.

After thresholding, a morphological erosion removes the remaining noise and false positives. However, this process also reduces the size of detected pathological areas, especially the thin or elongated parts, (see Fig. 4(D)), and the resulting regions are not always smooth and well-connected. The next part of the paper describes how to recover missing parts and obtain regularized results with an original segmentation method.

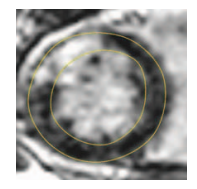

(A)

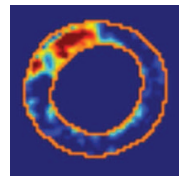

(B)

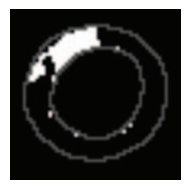

(C)

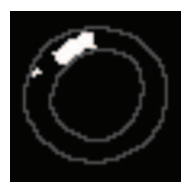

(D)
Fig. 4. (A) Original slice, (B) Fuzzy map, (C) 0.5 membership thresholding, (D) Morphological cleaning result. 


\subsection{Fast Region Competition}

In order to provide the abnormal tissue segmentation with additional spatial coherence, the previous statistical estimation of the intensity distributions is embedded into a regularized variational formulation. As a side contribution, we propose a novel algorithm to perform segmentation with a smoothness prior, specifically designed to be very fast with minimal complexity overhead compared to thresholding approaches.

Based on the the Region Competition framework [10], many variational two-phase segmentation methods consider the optimal partition as the best trade-off between smoothness and intra-class consistency. Over the set of all partitions $\mathcal{A}$ of a domain $\Omega$, an objective criterion is defined as the sum of a regularization term $\mathcal{R}(\mathcal{A})$ and application-dependent homogeneity measures $\left(r_{1}\right.$ and $\left.r_{2}\right)$ integrated over both the foreground and background regions of the image $I$ :

$$
\min _{\mathcal{A}}\left\{\mathcal{R}(\mathcal{A})+\int_{\mathcal{A}} r_{1}(I(\mathbf{x})) d \mathbf{x}+\int_{\Omega \backslash \mathcal{A}} r_{2}(I(\mathbf{x})) d \mathbf{x}\right\}
$$

Since the abnormal tissues can be of arbitrary topology, we use a level-set representation with an implicit function $\Phi$, positive in the foreground. $\Phi$ represents the foreground region $\mathcal{A}$, of characteristic function $H(\Phi)$ where $H$ is the Heaviside function. With this representation, (4) is equivalent to:

$$
\left.\min _{\Phi}\left\{\mathcal{R}(\Phi)+\int_{\Omega} H(\Phi(\mathbf{x})) r(\mathbf{x})\right) d \mathbf{x}\right\}
$$

with $r(\mathbf{x})=r_{1}(I(\mathbf{x}))-r_{2}(I(\mathbf{x}))$. To favor smooth results, the most classical additional regularization term $\mathcal{R}(\Phi)$ is the length of the boundary $\{\Phi=0\}$, which leads to an evolution equation that is driven by the curvature of the function $\Phi$. This has two shortcomings in practice: (a) the curvature term requires relatively slow iterative schemes to avoid instabilities; (b) weighting the two terms is critical and difficult to tune to get stable regularization effects on many images. We shall now describe our alternative formulation that has none of these drawbacks. Instead of ensuring smoothness by an additional term, the key idea to express the unknown partition as the super-level set of a smoothed function, which guarantees by construction that the boundary will indeed be smooth. For sake of simplicity and efficiency, we consider such smoothing to be the result of linear filtering, typically Gaussian. Let $w_{\sigma}$ be a symmetric non-negative low-pass kernel of scale $\sigma$, and

$$
\Phi_{\sigma}(\mathbf{x})=\left[w_{\sigma} * \Phi\right](\mathbf{x})=\int_{\Omega} w_{\sigma}(\mathbf{x}-\mathbf{y}) \Phi(\mathbf{y}) d \mathbf{y},
$$

the Fast Region Competition formulation is to solve:

$$
\left.\min _{\Phi \in[-1,1]}\left\{F(\Phi)=\int_{\Omega} H\left(\Phi_{\sigma}(\mathbf{x})\right) r(\mathbf{x})\right) d \mathbf{x}\right\}
$$

where the implicit representation of the region is the smooth function $\Phi_{\sigma}$ and the functional $F(\Phi)$ depends on the unknown function $\Phi$, before smoothing. $\Phi$ is further assumed bounded in $[-1,1]$ to remove the ambiguity induced by $F(\alpha \Phi)=F(\Phi)$ for any positive scalar $\alpha$. To solve (7), calculus of variation is applied to obtain the optimality condition for $\Phi$, when all directional derivatives vanish:

$$
\begin{gathered}
\forall \phi,\left.\quad \frac{\partial F(\Phi+h \phi)}{\partial h}\right|_{h=0}=0 \\
\forall \phi, \quad \int_{\Omega} \phi(\mathbf{y})\left\{\int_{\Omega} w_{\sigma}(\mathbf{x}-\mathbf{y}) \delta\left(\Phi_{\sigma}(\mathbf{x})\right) r(\mathbf{x}) d \mathbf{x}\right\} d \mathbf{y}=0 \\
\int_{\Omega} w_{\sigma}(\mathbf{x}-\mathbf{y}) \delta\left(\Phi_{\sigma}(\mathbf{x})\right) r(\mathbf{x}) d \mathbf{x}=0
\end{gathered}
$$

Since $w_{\sigma}$ is symmetric, the optimality condition (8) can be re-written as a convolution, using a continuous approximation $\delta_{\epsilon}$ of the Dirac distribution, i.e. $w_{\sigma} *\left[\delta_{\epsilon}\left(\Phi_{\sigma}\right) . r\right]=0$. Thus, a local minimizer of (7) can be found with a gradient-descent evolution equation of the form:

$$
\frac{\partial \Phi}{\partial t}=-w_{\sigma} *\left[\delta_{\epsilon}\left(\Phi_{\sigma}\right) \cdot r\right]
$$

This suggests a very simple iterative algorithm to perform regularized two-phase segmentation, with a single intuitive smoothness scale parameter $\sigma$, that does only require Gaussian convolutions: from an initial guess $\Phi^{0}$ at $k=0$ and for a given time step $\Delta t$, iterate until convergence:

- Compute $\Phi_{\sigma}^{k}$

- Update $\Phi^{k+1}=\min \left(\max \left(\Phi^{k}-\Delta t \cdot w_{\sigma} *\left[\delta_{\epsilon}\left(\Phi_{\sigma}^{k}\right) \cdot r\right],-1\right), 1\right)$

A natural choice for the homogeneity measures is to use maximum-loglikelihood criteria using the parameters estimated from the statistical analysis of myocardial abnormal $\left(\mu_{G}, \sigma_{G}\right)$ and healthy $\left(\sigma_{R}\right)$ tissues:

$$
\begin{aligned}
& r_{1}(I)=-\lambda_{1} \log \left(p_{G}\left(I \mid \mu_{G} ; \sigma_{G}\right)\right) \\
& r_{2}(I)=-\lambda_{2} \log \left(p_{R}\left(I \mid \sigma_{R}\right)\right)
\end{aligned}
$$

$\lambda_{1}$ and $\lambda_{2}$ can usually be fixed to 1 . However, in our experiments, a slightly higher weight is assigned to healthy tissues $\left(\lambda_{1}=0.7\right.$ and $\left.\lambda_{2}=1.3\right)$. This bias offers additional control by favoring an healthy membership for pixels whose posterior probability is not very discriminative, and gives better results in the case of partial volume effect or when there is no pathological image evidence (single distribution).

\section{QUANTIFICATION RESULTS}

The method was tested on 11 short-axis LECMR volumes (100 slices) acquired in a multi-center study between 2004 and 2007 (Philips Intera scanner 1.5T, Fast Field Echo sequence, $\mathrm{TE}=1.1 \mathrm{~ms}, \mathrm{TR}=3.8 \mathrm{~ms}$, flip angle $=15$ ). All slices are $256 \times 256$, spaced with $10 \mathrm{~mm}$ and with pixel size around 
$1.5 \mathrm{~mm}$. For various enhancement patterns, the segmentation results are satisfactory and obtained in around 0.2 second per slice. See Fig. 5 for illustrations.

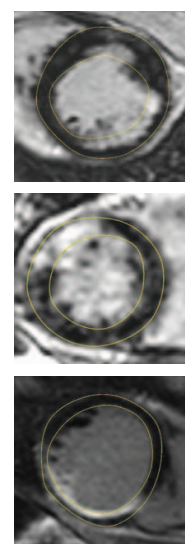

(A)
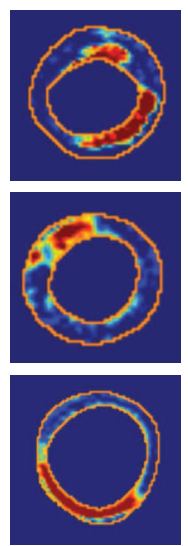

(B)
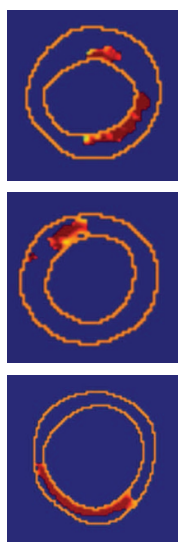

(C)
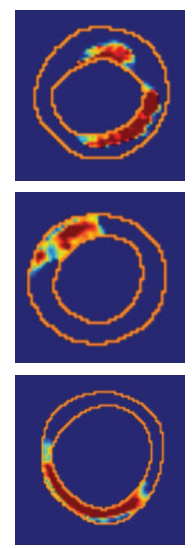

(D)
Fig. 5. Detection examples: (A) original slice, (B) corresponding fuzzy map, (C) cleaning result, (D) Final segmentation result overlayed with fuzzy map.

In order to validate our method, we quantify the segmented areas and derive clinically relevant parameters. We focus on two principal characteristics: the percentage of abnormal tissues and the transmurality or extent of abnormal tissue in the radial direction.

To evaluate the percentage of pathological tissues, we divide the myocardium in angular sectors, in which we compute the average of detected voxels membership to the abnormal class. The transmurality is computed from a normalized representation independent of both myocardium shape and size. First the distance of the segmented regions to the endocardium and epicardium contours are computed and normalized. The transmurality error is expressed as a percentage with respect to myocardium thickness.

The tested database was studied by an experienced observer, who manually segmented pathological regions with the interactive thresholding procedure offered in the Philips ViewForum 4.3 MR Cardiac Package. 100 slices are available with manual references, used to evaluate the proposed method, as well as two classical thresholding approaches, noted 2SD th. and 3SD th., which consist of thresholding the myocardium intensity respectively at 2 and 3 standard deviations above the average healthy myocardium intensity. As shown in Tab. 1, our method shows better agreement with the expert segmentations than thresholding approaches, though the ground truth was defined with manual thresholding.

Moreover for all tested slices, including images without apparent pathological tissues, our method avoids false positive detections and provides spatially coherent results, contrary to intensity-based thresholdings.

\begin{tabular}{|c|c|c|c|}
\hline Measure & Our method & 2SD th. & 3SD th. \\
\hline \hline $\begin{array}{c}\text { Volumic error } \\
\text { (\% w.r.t. heart volume) }\end{array}$ & $3.6 \pm 5.6$ & $4.7 \pm 6.1$ & $4.6 \pm 7.7$ \\
\hline $\begin{array}{c}\text { Transmur. error (\% w.r.t. } \\
\text { myocardium thickness) }\end{array}$ & $12 \pm 11$ & $12 \pm 10$ & $14 \pm 10$ \\
\hline
\end{tabular}

Table 1. Comparison of automatic method and manual detection of pathological areas.

\section{CONCLUSION}

We proposed a novel fully automatic method for myocardium pathological tissues segmentation and provided quantification parameters allowing a reliable viability assessment. Experiments were promising and have demonstrated the robustness and accuracy of the segmentation when compared with manual detections. Future work will be based on fully 3D MR scans to improve the results with $3 \mathrm{D}$ connectivity analysis.

\section{REFERENCES}

[1] R.J. Kim et al., "Relationship of MRI delayed contrast enhancement to irreversible injury, infarct age, and contractile function," Circulation, vol. 100, no. 19, pp. 1992-2002, 1999.

[2] A.T. Yan et al., "Characterization of the peri-infarct zone by contrast-enhanced cardiac magnetic resonance imaging is a powerful predictor of post-myocardial infarction mortality," Circulation, vol. 114, no. 1, pp. 32, 2006.

[3] A. Schmidt et al., "Infarct tissue heterogeneity by magnetic resonance imaging identifies enhanced cardiac arrhythmia susceptibility in patients with left ventricular dysfunction," Circulation, vol. 115, no. 15, pp. 2006, 2007.

[4] L.Y. Hsu et al., "Quantitative myocardial infarction on delayed enhancement MRI. part II: Clinical application of an automated feature analysis and combined thresholding infarct sizing algorithm," J. Magn. Reson. Imag., vol. 23, no. 3, 2006.

[5] E. Heiberg et al., "Semi-automatic quantification of myocardial infarction from delayed contrast enhanced magnetic resonance imaging," Scandinavian Cardiovascular J., vol. 39, no. 5, pp. 267-275, 2005.

[6] A. Hennemuth et al., "A comprehensive approach to the analysis of contrast enhanced cardiac MR images," IEEE Trans. Med. Imag., vol. 27, no. 11, 2008.

[7] C. Ciofolo et al., "Automatic myocardium segmentation in late-enhancement MRI," in Proceedings of ISBI'08, 2008, pp. 225-228.

[8] H. Gudbjartsson and S. Patz, "The Rician distribution of noisy MRI data," Magn. Reson.Med., vol. 34, no. 6, pp. 910, 1995.

[9] AP Dempster et al., "Maximum likelihood from incomplete data via the EM algorithm," J. Roy. Stat. Soc. Series B (Method.), pp. 1-38, 1977.

[10] S.C. Zhu and A. Yuille, "Region competition," IEEE Trans. Pattern Anal. Mach. Intell., vol. 18, no. 9, pp. 884-900, 1996. 